\title{
Lockdown Anthropology and Online Surveys: Unprecedented Methods for Unprecedented Times
}

Nicholas J. Long

\begin{abstract}
This paper explores the value of online surveys as a methodology for conducting anthropological research on, and during, lockdowns implemented to curb the transmission of SARS-CoV-2, the coronavirus responsible for COVID-19. I argue that despite longstanding disciplinary suspicion towards survey methods, qualitative online surveys may be more useful than conventional ethnography for developing the analytical frameworks necessary to comprehend the social realities that are emerging as a result of large-scale social distancing regimes.
\end{abstract}

As SARS-CoV-2 spreads across the world, it has become commonplace to suggest we live in unprecedented times. Certainly, as an anthropologist who has long worked on emergent socialities and citizens' engagements with regimes of governmentality, the pandemic presented me with an unprecedented predicament. I was eager to investigate the new social worlds instigated by the pandemic and contribute to the academic and political debates surrounding them, yet my go-to methodology of ethnographic fieldwork was impossible 
given lockdown regulations. And so I did something that was-for me, at least-equally unprecedented. I set up an online survey.

\section{Surveys and Anthropology: An Awkward Relationship}

Before 2020, my disposition towards surveys had been decidedly chilly. Anthropologists, after all, pride themselves on using long-term ethnographic fieldwork to develop depths of insight survey research is thought to lack. My own work, for instance, has critiqued Barometer surveys for capturing conventionalised expressions of dissatisfaction with democracy, rather than illuminating the intersubjective origins of democratic malaise (Long 2016). Consequently, as Datta and Vaid $(2018,142)$ note, when anthropologists do use surveys, these generally stay subordinate to ethnography in the analytical process. They might be used to 'quantitatively verify' hypotheses developed through ethnography (Snodgrass et al. 2016, 60), or to provide an initial overview of the range of experiences within a population, contextualising the 'rich narratives' elicited in follow-up ethnographic interviews (O'Connor 2019, 249). One of the few recent articles in an AAA journal based primarily on survey data offers a more positive vision, noting online surveys 'give access to a wider and more diverse array of respondents' and 'uniquely permit both qualitative and quantitative insights' (Phillips and Rees 2018, 220). However, by stressing the need for such research to be 'embedded in deep ethnographic experience' $(2018,219)$, even that article positions ethnography as the preferred tool with which to develop one's primary understanding of a social world.

While ethnography's strengths cannot be denied, disciplinary chauvinism towards surveys risks overlooking their value as a research tool, especially when fieldwork proves impractical. Understanding how professional anthropologists of colour experience university life would be nigh-on impossible to study ethnographically without compromising 
confidentiality; Brodkin et al. (2011) nevertheless found an online survey on the topic afforded important and anthropologically robust findings and policy recommendations. Sometimes, then, a survey can be good enough. But I want to push the argument further, arguing that, when dealing with a situation as unprecedented as the COVID-19 pandemic, it is surveys, not ethnography, that can best provide a conceptual framework for understanding emergent social realities.

\section{My Survey Journey}

My first ever survey arose from my frustrations with the rigid lockdown measures adopted by the UK government in March 2020. These required the public to 'stay at home', severing the networks of support and care on which many households depend, and plunging millions of single-person households into isolation (Long 2020). By contrast, New Zealand had allowed those living alone or with complex childcare needs to pair up with 'buddies' to form multihousehold 'bubbles': exclusive social networks that would provide opportunities for support and social contact whilst limiting exposure to - and containing the spread of - coronavirus. Hoping to translate anthropological ideas about the primacy of relationality to a policy audience, I designed a survey to test the hypothesis that buddying arrangements enhanced experiences of lockdown. This survey was, primarily, a quantitative verification device; I didn't yet see it as a tool with the range or power of ethnography.

That changed when declining case numbers led the New Zealand government to announce that everyone would be allowed to form small, exclusive, multi-household bubbles as the nation entered 'Level 3' on April 28th. Concomitantly, 'social bubbles' schemes began attracting interest in Britain. Yet they also raised questions and concerns: How widespread was uptake likely to be? Would bubbles stay exclusive? Might dilemmas over who to bubble 
with tear relationships apart? Nobody could answer these questions: never before had there been a society of bubbles.

But such a society was about to emerge in New Zealand. Suddenly I saw new potential in my survey. No longer need analysis focus on those eligible for buddies, with others serving as 'control' data. Every response would now illuminate how and why a bubble policy was engaged with. New questions were added, a social media ad campaign designed. It proved so successful that I designed five further surveys (two for New Zealand, three for the UK), attracting over 8,800 responses to date.

\section{What I Have Learned}

Online surveys are remarkably agile and responsive research tools. They can be put online within hours of a research question arising or a policy change being announced, quickly gathering hundreds of responses. They are also an efficient way of documenting evanescent social worlds that stand to disappear as lockdown measures are lifted, amended, or reimposed. Their reach of course has limits, excluding those without sufficient time, linguistic fluency, internet access, or indeed appetite to participate. Survey research in New Zealand has historically attracted disproportionately high numbers of women and university graduates, and comparatively few Māori (Houkamau and Sibley 2019); my own surveys were no exception, and comparable skews were observable in the UK study. These limitations can, however, be partly offset - by targeting adverts to underrepresented demographics, disseminating the survey via pre-existing research networks and community organisations, running the survey in multiple languages, or engaging research assistants or 'citizen scientists' to work with hard-to-reach populations. Even if still not strictly representative, the resulting dataset will nevertheless afford substantive insights into diverse ways of life. 
And survey data can be extraordinarily rich. Juxtaposing factual questions about what respondents had done or felt with free-text boxes where they could elaborate on their responses, my surveys illuminated broad patterns of practice whilst soliciting multi-faceted and nuanced accounts of life under lockdown. Responses displayed considerable candour and few signs of the conventionalization that bedevils Barometer surveys-perhaps because questions focused on specific relationships and events, encouraging personal storytelling, perhaps because lockdown was itself such an extraordinary experience, one that simultaneously invited and provided time for deep reflection.

Many important anthropological insights have arisen in consequence. The New Zealand surveys, for instance, provided a valuable overview of whether, how and to what effect social networks were remaking themselves under the social bubbles policy (see Long et al. 2020). If this echoes one of the most old-fashioned goals of ethnographic fieldwork - to provide descriptive accounts of 'social structure' - it also affords timely and policy-relevant findings. Government messaging that emphasised bubbles as a support mechanism simplified dilemmas of bubble membership; respondents bubbled with those in most need. Fear of the coronavirus led respondents to avoid bubbling unless absolutely necessary. However, those seen as infection risks (e.g. frontline workers) and those with few social contacts (e.g. arriving migrants) struggled to access support. They remained isolated.

Filtering survey responses by reported life satisfaction, contentment with bubble arrangements, and other quantitative or qualitative measures of subjective wellbeing, revealed further circumstances disproportionately associated with certain experiences of lockdown. While self-proclaimed introverts enjoyed the enforced isolation, for instance, others were struggling. Parents sharing childcare with an ex often felt aggrieved at their forced incorporation into that ex's own bubble expansion. The recently bereaved struggled with their grief in the absence of mutual physical consolation. In the UK surveys, widow/ers 
trapped at home alone were consumed by memories of their partners, and, like other lone household dwellers, angry that the government had not allowed them New Zealand-style bubbles before the $12^{\text {th }}$ June. As this demonstrates, lockdown experiences do not just affect wellbeing, they also shape political subjectivities.

Reading through thousands of survey narratives, patterns became apparent that may have been submerged in an overly quantitative survey, but would have emerged much more slowly - if at all - via ostensibly 'richer' methods such as ethnographic interviews. This matters, because the axes of relational and circumstantial disadvantage the surveys identified are notably distinct from conventional categories of structural disadvantage, or the 'populations of interest' identified as priorities for pandemic mental health research (Holmes et al. 2020). Many have only become significant disadvantages in the context of the pandemic and associated social distancing regimes. The unprecedented nature of the COVID19 crisis has generated unprecedented new forms of vulnerability and, by extension, new topologies for both social analysis and for activism - although these vary between different national or regional coronavirus responses. Established analytics of intersectionality must thus be updated, and fast. That requires working from the ground up, mapping the circumstances, categories and concerns that meaningfully structure people's experiences-as good anthropologists always have done. Survey research offers a powerful and timely way of doing this within the unprecedented social worlds in which we now live.

\section{References}

Brodkin, K., Morgen, S., and Hutchinson, J.. 2011. Anthropology as White Public Space? American Anthropologist 113(4), 545-556.

Datta, A., and Vaid, D. 2018. Mind the Gap? Navigating the Quantitative and the Qualitative in Survey Research. Studies in Indian Politics 6(1), 140-145. 
Holmes, E.A., O’Connor, R.C., Perry, V.H., et al. 2020. Multidisciplinary Research Priorities for the COVID-19 Pandemic: A Call for Action for Mental Health Science. Lancet Psychiatry 7(6), P547-P560.

Houkamau, C.A., and Sibley, C.G. 2019. The Role of Culture and Identity for Economic Values: A Quantitative Study of Māori Attitudes. Journal of the Royal Society of New Zealand 49(S1), 119-136.

Long, N.J. 2016. Why Indonesians Turn against Democracy. In The State We're In: Reflections on Democracy's Troubles (eds) J. Cook, N. J. Long and H. L. Moore, 7196. Oxford: Berghahn. 2020. From Social Distancing to Social Containment: Reimagining Sociality for the Coronavirus Pandemic. Under review.

Long, N.J., Aikman, P.J., Appleton, N.S. et al. 2020. Living in Bubbles During the Coronavirus Pandemic: Insights from New Zealand.

$<$ http://eprints.lse.ac.uk/104421/>

O’Connor, B.H. 2019. "Everything Went Boom”: Kinship Narratives of Transfronterizo University Students. The Journal of Latin American and Caribbean Anthropology 24(1), 242-262.

Phillips, T., and Rees, T. 2018. (In)Visibility Online: The Benefits of Online Patient Forums for People with a Hidden Illness: The Case of Multiple Chemical Sensitivity (MCS). Medical Anthropology Quarterly 32(2), 214-232.

Snodgrass, J.G., Lacy, M.G., Denagh, H.J.F, et al. 2016. Culture and the Jitters: Guild Affiliation and Online Gaming Eustress/Distress. Ethos 44(1), 50-78. 